\title{
Computational fluid dynamics modelling of heat treatment of single crystal nickel based superalloys for turbine blade application
}

\author{
Francesco Cosentino ${ }^{1}$, Nils Warnken ${ }^{1}$, Jean-Christophe Gebelin ${ }^{1}$, \\ Robert W Broomfield ${ }^{1}$ and Roger C Reed ${ }^{1}$ \\ ${ }^{1}$ Department of Metallurgy and Materials, The University of Birmingham, \\ Edgbaston, Birmingham, B15 2TT, UK
}

Keywords: Heat Treatment, CFD modelling, RR3010, microstructure, creep.

\begin{abstract}
A numerical model based on computational fluid dynamics $(\mathrm{CFD})$ is developed to simulate the vacuum heat treatment of single crystal turbine blades. Radiation and forced convection heat transfer are taken into account to model ramping-up, holding and gas fan quenching. This enables quantitative predictions to be made of temperature fields inside a laboratoryscale vacuum furnace. The uniformity of the expected temperature field is studied with a particular aim to predict hot spots or locations with higher risk of incipient melting. Simulation of the gas fan quenching process allows estimation of the quench rate to be made as a function of position within the furnace and critical furnace processing parameters. The effect of quench rate and ageing time on the microstructure of RR3010 superalloy has been characterised using scanning electron microscopy. Two representative structures with different average $\gamma^{\prime}$ size have been tested in creep in three temperature regimes and with three different level of stresses, in an attempt to gain better understanding of the influence of microstructure on creep performance. Our results indicate that at lower creep testing temperatures, larger $\gamma^{\prime}$ size confers longer time to rupture; however, as the testing temperature increases, the influence of microstructure is less pronounced.
\end{abstract}

\section{Introduction}

Single crystal nickel based superalloys used for turbine blade application are manufactured by investment casting. The conditions used during processing produce a casting with a high degree of microsegregation. Thus, the distribution of elements in the as-cast state is not uniform with chemical heterogeneity present on a scale inherited from the dendritic microstructure formed during solidification, see Fig. 1. The presence of such microsegregation is known to impair the mechanical properties considerably and therefore, during the manufacturing process, a heat treatment stage is often employed [1]. This involves a slow ramping up of the temperature, followed by a holding at a temperature above the $\gamma^{\prime}$ solvus and finally a fast quench, during which a fine dispersion of precipitates is produced. The heating rate should be slow enough to avoid incipient melting, while the temperature and the duration of the holding should be enough to dissolve interdendritic $\gamma^{\prime}$ precipitates. During quenching a particular concern is the uniformity of cooling rates between components produced in any given batch. It is evident that this is a complicated process for which the precise control of the temperature plays a vital role. So far, very little attention has been paid to the mathematical modelling of the heat treatment process, perhaps due to its inherent complexity. Currently the analysis and optimisation of the heat treatment process relies heavily on trial-and-error experimentation, even though numerical simulation capabilities are becoming more readily available. In

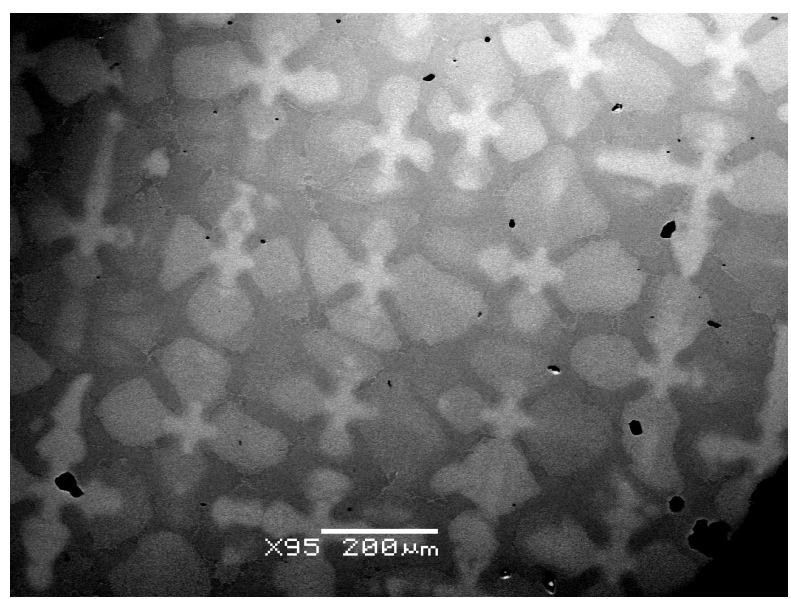

Figure 1: Scanning electron micrograph of an as-cast RR3010 turbine blade showing a typical dendritic microstructure. 
this work, a model based on computational fluid dynamics (CFD) is presented for the simulation of the heat treatment of turbine blades. Our modelling focusses on a laboratory-scale vacuum furnace and takes into account the radiation heat transfer during the heating stage and the forced convection heat transfer commonly used during cooling. The model provides prediction of the thermal history of the treated component and can be used to identify the regions that are at risk of incipient melting. Simulation of the gas flow provides prediction of cooling rates in different regions of the blade aerofoil and as a function of the position in the furnace load. These results can be used to assess the uniformity of quenching, and to improve the furnace design towards a more homogenous flow field.

Furthermore, it is very important to correlate the effect of heat transfer to microstructure and properties of the alloy. Microstructure models exist that, taking into account the thermodynamic of the alloy and transformation kinetics, are able to predict microstructural features like dendritic arrays and evolution of microsegregation [2] or precipitation and coarsening of the $\gamma^{\prime}$ phase $[3,4]$. Naturally, these models need processing information, i.e. temperature histories of the type that the present modelling can provide, so that one ambitious goal is the coupling of processing and materials models so that properties can be predicted as a function of processing data. For the heat treatment process, the ultimate goal is to improve material properties and in particular the creep life. There is undoubtedly an influence of microstructure and thus cooling rate; therefore it is important to understand how the microstructure influences creep life in order to optimise the performance of any existing alloy. Therefore, in this paper extensive characterisation of the influence of cooling rates and ageing times upon the development of the $\gamma-\gamma^{\prime}$ structure has been performed. Based on the results, two microstructures were selected for evaluation of their mechanical performance. Creep testing was performed, at three test temperatures and three stresses per temperature.

\section{Numerical Modelling}

In this work, computational fluid dynamics (CFD) methods are employed. It is well known that CFD methods are well established for treating a wide variety of engineering problems particularly involving heat transfer and fluid flow; however, it does not appear to have been used widely for furnace modelling so far. In this research CFD has been used to model a vacuum heat treatment furnace using the commercial software package ANSYS Fluent version 13. During the ramping up stage, heat is transferred by radiation in a very low pressure atmosphere and therefore natural convection is not significant; in this investigation the mechanisms of heat transfer considered are radiation between surfaces and conduction in solids. Cooling is obtained by high pressure gas quenching. In order to achieve this, the furnace is pressurized to a predefined level and a fan used to drive the flow through the furnace chamber. In this latter case, the main mechanism of heat transfer is forced convection; thus radiation has not been taken into account. Due to the different physical processes involved, the simulation activity has been divided into two separate stages.

\section{Modelling of the heating stage}

The geometrical model used for the heating stage is illustrated in Fig. 2 together with the furnace used for the validatory experimentation. The model was designed using CAD files of the furnace geometry, which is located in the Interdisciplinary Research Centre (IRC) at the University of Birmingham; although of a laboratory scale, it possesses most of the characteristics of a industry-scale gas fan quenching (GFQ) furnace. Initially, our modelling has dealt with three cylindrical specimens placed side by side, the rack used to accommodate the furnace load and a set of heating elements, all enclosed in a cubic chamber made out of insulating material; this is further bounded by the external vessel, water cooled to room temperature. The model was then further developed to include a turbine blade of realistic geometry. With this model, transient calculation were performed to predict temperature fields inside the furnace during the heat treatment.

One overarching requirement for realistic modelling is the specification of the heat balance in the furnace. This was accomplished by specifying the temperatures of the heating elements as a function of time and assuming these to be uniform. The boundary conditions used for the simulated heat treatments are given in Fig. 3. The heat losses from the furnace walls were assumed to be due to radiation alone, and the external far field temperature was set to $300 \mathrm{~K}$. Natural convection was neglected in the calculation. Radiation was accounted for using the S2S radiation model as implemented in ANSYS Fluent [5]. The S2S radiation model considers the radiation from surface to surface; it does not consider any influence of 


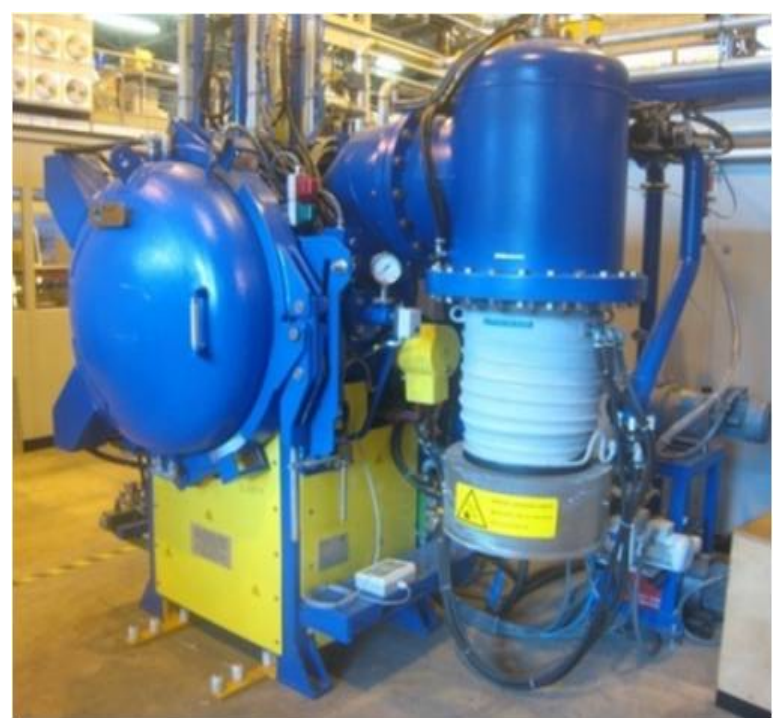

(a) Laboratory-scale vacuum furnace at the University of Birmingham.

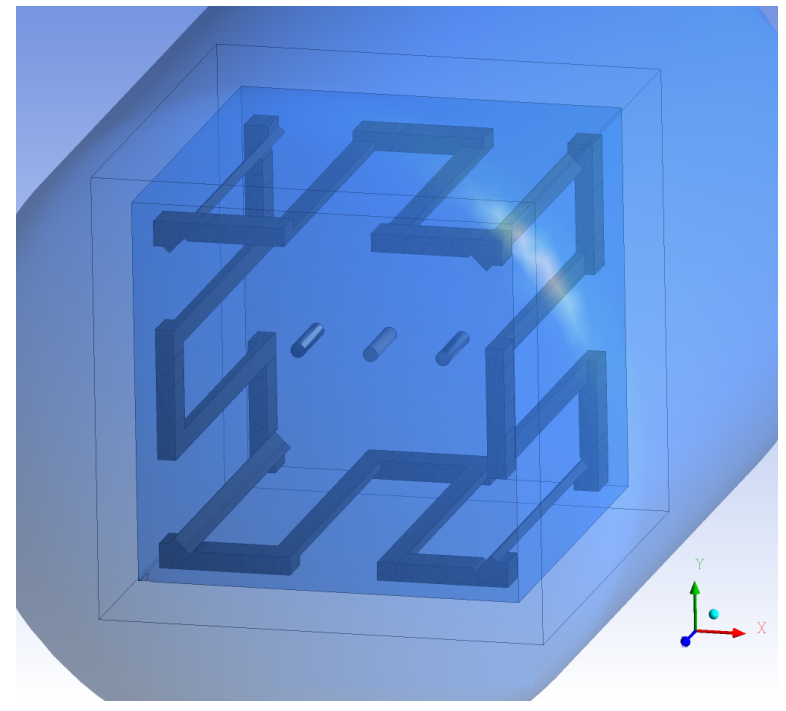

(b) Computer model of the furnace arrangement to be simulated.

Figure 2: Vacuum furnace used for validation experiments and associated CAD geometry.

the gas phase such as scattering or absorption. The heat flux emitted from a surface is proportional to its temperature and to a combination of size, orientation and distance of all surfaces with respect to each other. All these geometric features are accounted for using appropriate viewfactors.

\section{Modelling of the gas quenching}

Modelling of the cooling process requires the computation of the flow field inside the furnace chamber. Computational fluid dynamics [6] has been used to solve numerically the Navier-Stokes equations, coupled with the heat transport equations, using a finite volume approach. This gives the time dependent temperature within the solution domain. The effect of turbulence has been included using the $k$ - $\omega$ model [5]. The boundary conditions for the flow simulation were specified in terms of the pressure drop between the inlet and the outlet, assuming uniform pressure distribution at each boundary. For the specification of these values, measurements were performed using a differential pressure transmitter. The furnace parameters that control the intensity of the quenching are the gas pressure and the percentage of the maximum fan speed. For any given combination of these used in a validation experiment, the pressure difference between the inlet and outlet was measured and applied in the calculation. In order to keep the runtime at an acceptable level, the physics of the problem has been simplified. It has been assumed that the main mechanism of heat transfer is forced convection; therefore the contributions of radiation heat transfer and natural convection are not been included in the calculations. Moreover it is assumed that the flow field does not change with temperature. The geometry of the furnace used for quenching simulations is different from that of the heating model; here the model includes openings that allow the gas to enter and leave the furnace (i.e. inlet and outlets) as well as baffles that are of primary importance in determining the overall flow field.

\section{Simulation Results and Experimental Validation}

\section{Heating model}

Transient simulations were performed to predict the heating curves of (i) cylindrical specimens and (i) a representative turbine blade. The temperature predicted by the model was validated against thermocouple readings. The $1.5 \mathrm{~mm}$ diameter thermocouples (type N) were positioned inside holes drilled along the centreline of the cylindrical specimen to a typical depth of $4 \mathrm{~mm}$. Experiments were repeated to ensure consistent reading and the measurement were found to be within the limits of error of the instrument ( $0.75 \%$ of the reading). In Fig. 4 the furnace temperature is shown at the holding temperature. The 


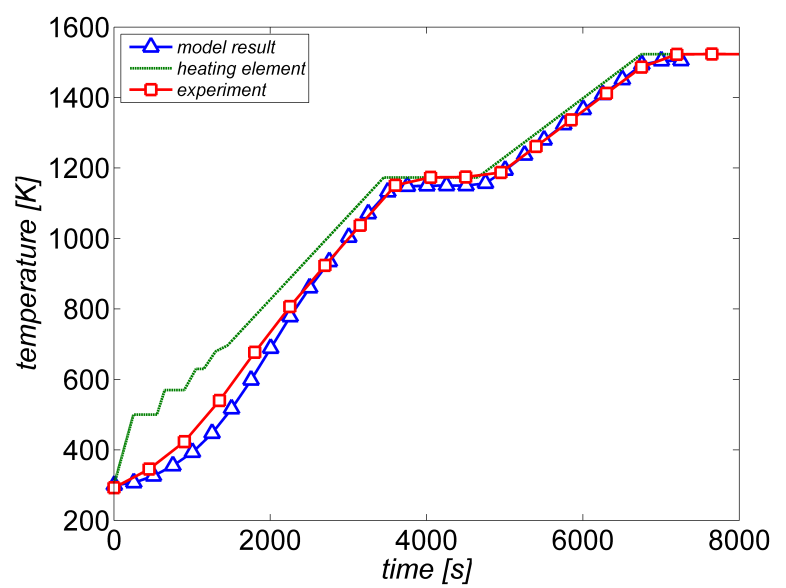

(a) First attempt.

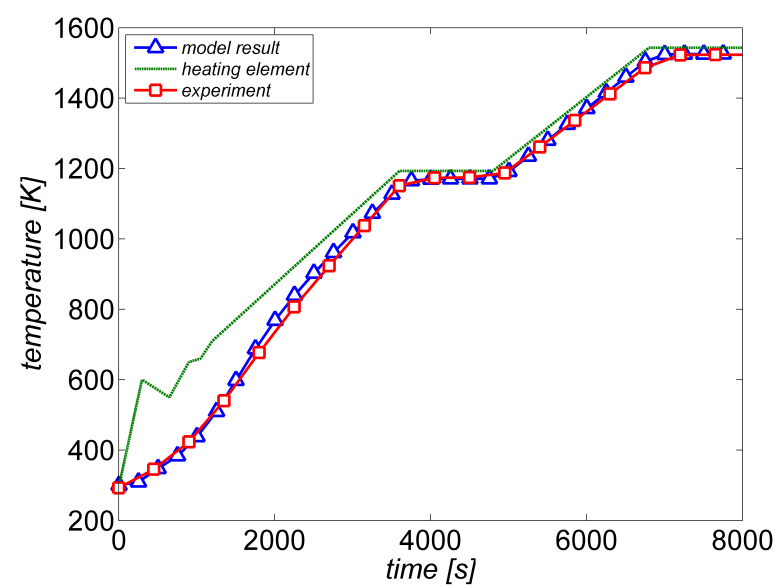

(b) Refined boundary condition.

Figure 3: Simulation result. The plots show the temperature as a function of time. The curve with square marker represents the measured experimental value; the curve with no marker is the simulation boundary condition and the triangle marked is the model prediction.

heating element temperature is uniform, consistent with its use as a boundary condition. The temperature decreases towards the centre of the furnace but the higher gradients are found between the heating elements and the walls, where temperature reaches a minimum value. In the core of the furnace (the space occupied by rack and samples) the temperature distribution is influenced by the presence of the rack which shadows the heater radiation, and the gradient is much more shallow with a variation in temperature is about $6 \mathrm{~K}$ at the holding, which can be considered as uniform. Of course during the ramping this situation is worse. Due to symmetry, the temperatures of the cylinders on either side are almost identical, and higher than the temperature of the central cylinder by half a degree. Although not significant in this case it is important to be able to quantify this temperature difference since it can become significant in industrial scale applications where an elevated number of parts are heat treated at the same time. Fig. 3-a shows the temperature of a point inside the middle cylinder and compares it with the predictions of the model. Good agreement is observed especially at higher temperatures, whilst at lower temperatures the cylinder temperature is underpredicted. There are several possible reasons for this discrepancy, but the main source of error is considered to be the accuracy of the boundary condition. The temperature of heating elements cannot be directly measured using thermocouples and because of the unsteadiness of the heating process it is not trivial to derive. Refinement of the boundary condition therefore remarkably improves the simulation accuracy (as shown in Fig. 3-b). The temperature distribution on the surface of the heat treated components was found to vary considerably depending on the stage of the heat treatment. This is illustrated in Fig. 5 for the case of the turbine blade considered. In Fig. 5-a the temperature during holding is illustrated. Here the temperature of the heating element is fixed; the overall temperature variation in

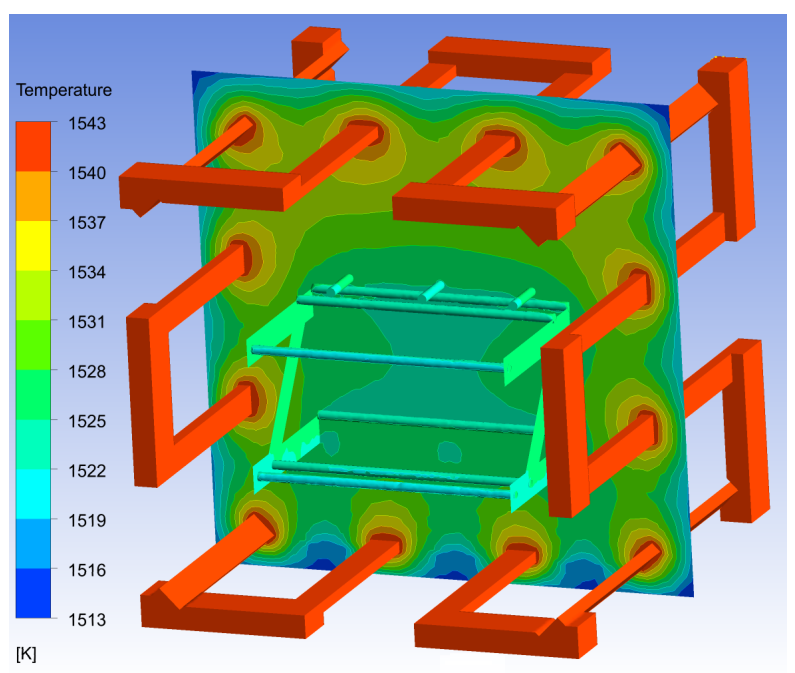

Figure 4: Prediction of temperature distribution during holding. 


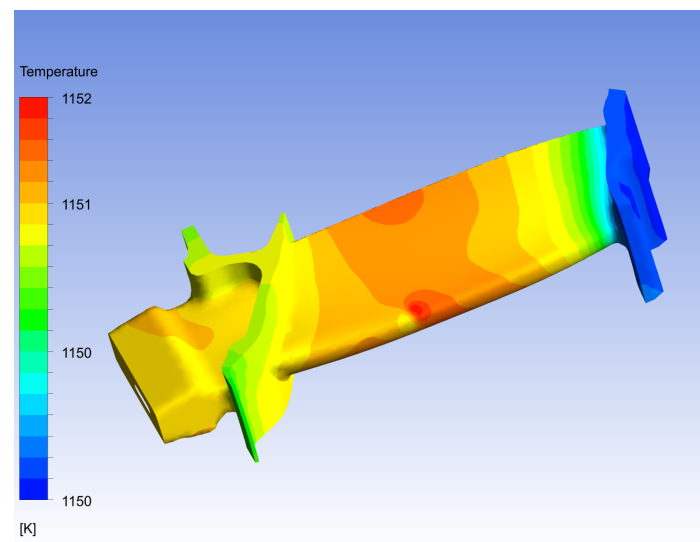

(a) snapshot during holding

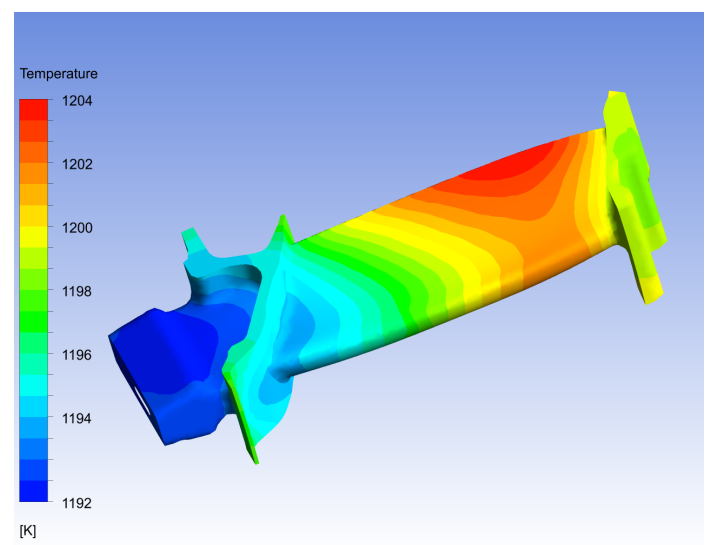

(b) snapshot during ramping up

Figure 5: Temperature distribution on the surface of a turbine blade. Isothermal hold (a) and ramping (b).

the blade is predicted to be $2 \mathrm{~K}$. The temperature is highest at mid-height of the aerofoil. The minimum is located at the shroud which is not facing directly the heating element. The root is equally not facing the heating element, but the higher mass provides a higher thermal inertia and hence a lower temperature. During the transient stage the temperature difference is found to be greater, and can be larger than $10 \mathrm{~K}$, as shown in Fig. 5-b. Here the temperature is higher on the aerofoil and lower in the root, indicating that during heating the temperature is a function of the local thickness of the component.

\section{Quenching model}

Calculation of fluid flow during quenching was performed to predict cooling rates of the treated components. In order to get accurate results, the flow quantities at the boundaries must be defined with care. Since both velocity and pressure are not uniform, specifying constant quantities at the boundaries in the model of Fig. 6-a resulted in velocity vectors normal to the inlet, which did not capture the flow inhomogeneities. It was then necessary to simulate how the gas enters into the furnace by moving the inlet backwards and by including the top duct into the model, as shown in Fig. 6-b. In this way the flow develops in the top channel so that the gas direction at the entrance of the hot zone is correctly reproduced. Simulations of different quenching conditions were performed. In what follows, the results of quenching at 1 bar of absolute pressure and $30 \%$ fan speed where the maximum fan speed is 3000 revolutions per minute $(\mathrm{RPM})$ - are discussed. These conditions resulted in a pressure drop across the furnace of about $30 \mathrm{~Pa}$. Fig. 6 -b shows a side view of the furnace. The flow is entering the domain from the inlet - note that the fan itself has not been explicitly modelled - and travels through the furnace leaving from the outlet. The flow velocity at the inlet is about $2.5 \mathrm{~m} / \mathrm{s}$, for the combination of pressure and fan speed mentioned earlier. It then accelerates near the entrance of the hot zone because the section is smaller. The highest velocity is found above the first baffle and between the last two baffles, which is where the flow starts to change direction to enter the hot zone. The position and orientation of the baffles direct the flow towards the back of the furnace, hence the quenching is expected to be inhomogeneous. The velocity magnitude of the flow hitting the blade varies between 1 and $3 \mathrm{~m} / \mathrm{s}$. At the furnace exit, similar to the entrance, there is an increase of flow speed because the section is smaller. Fig. 6-c shows the velocity magnitude in a front view of the furnace. Due to the symmetry of the furnace the flow field is symmetric as well, and flow separation can be observed due to the presence of the heating elements. The cooling curve predicted from the simulation agrees well with the experimental value measured using a thermocouple fitted in a hole inside a cylinder, as shown in Fig. 7. The agreement is good considering that radiation and natural convection have been neglected. In particular, the influence of pressure and fan speed is captured in a realistic way. The flow field depicted in Fig. 6-b and -c is also validated by the readings of four retractible thermocouples which are placed near the four corners of the furnace. For each of the quenching experiments, the two thermocouples in the back corners read higher 


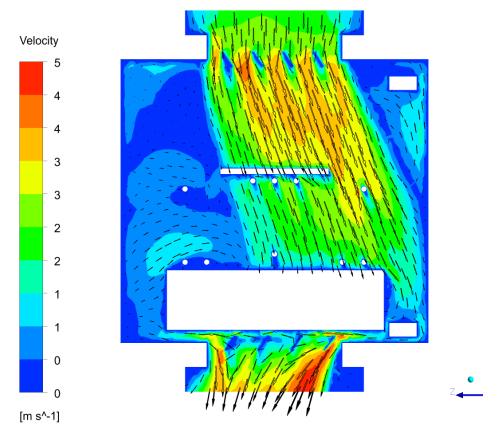

(a) Initial geometry

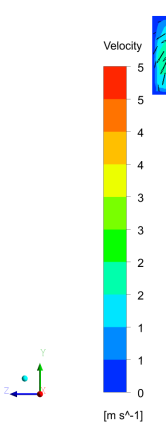

(b) Final geometry: side view
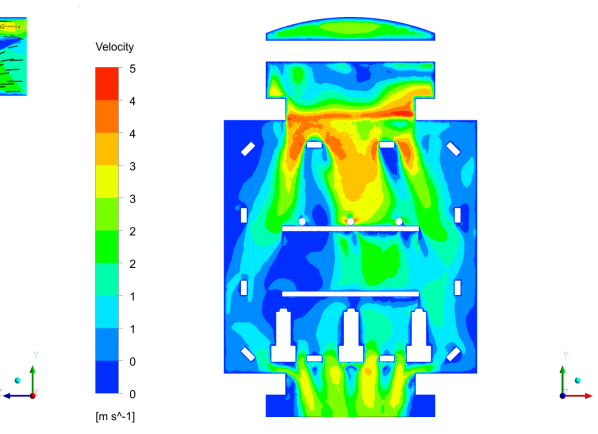

(c) Final geometry: front view

Figure 6: Quenching furnace model: Velocity magnitude profiles for different geometries.

cooling rates than the ones in the front, consistent with a higher flow speed at the back of the furnace. The influence of the flow field can also be observed in terms of their effect on heat transfer and temperature on the quenched components. Fig. 8 shows the calculated heat transfer coefficient (HTC) distribution on the surface of the blade. The side of the blade that faces the incoming flow shows higher values of HTC than the back and the root, which was oriented towards the back part of the furnace. The temperature on the other hand is influenced by a combination of HTC and local thickness of the component, as can be seen in Fig. 9. The temperature is higher in the root, despite the greater HTC there, because of the greater mass; for the same reason (very low mass)

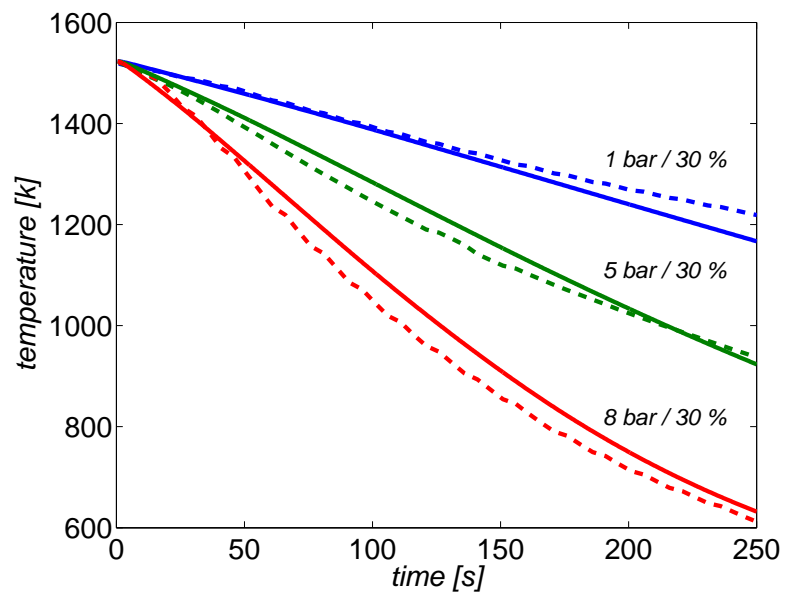

Figure 7: Quenching curves for different pressure and fan speeds. Comparison between experiment (dashed line) and simulation results (solid line). the aerofoil is rapidly quenched. The temperature difference across the entire blade is quite large, which leads to very different local cooling rate.

\section{Microstructure characterisation}

The microstructure of the single crystal superalloy RR3010, subjected to different quenching conditions and ageing times, has been characterised using scanning electron microscopy (SEM). Solution heattreated cylindrical specimens were subjected to solutioning treatments of 1.5 hours at $1637 \mathrm{~K}\left(1364^{\circ} \mathrm{C}\right)$. After the dissolution of $\gamma^{\prime}$, the samples were quenched at four different cooling rates, followed by ageing for three different times at $1423 \mathrm{~K}\left(1150{ }^{\circ} \mathrm{C}\right)$. The details are summarised in Table 1.

The characterisation focussed on the determination

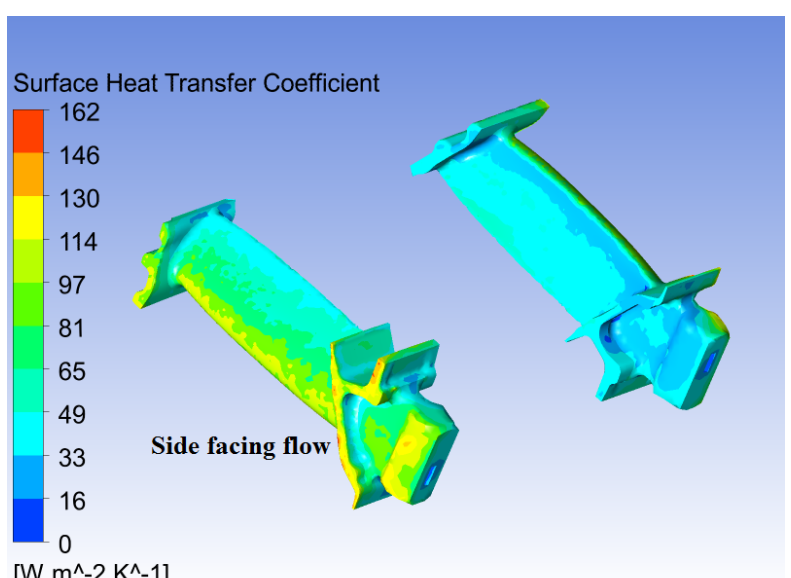

$\left[\mathrm{W} \mathrm{m}^{\wedge}-2 \mathrm{~K}^{\wedge}-1\right]$

Figure 8: Variation of heat transfer coefficient on the blade surface. 
Table 1: Quenching plus ageing conditions matrix

\begin{tabular}{|c|ccc|}
\hline Measured Cooling Rate & \multicolumn{3}{|c|}{ Ageing at $1423 \mathrm{~K}$} \\
\hline $251 \mathrm{~K} /$ minute & 1 hour & - & 5 hour \\
$362 \mathrm{~K} /$ minute & 1 hour & 3 hour & 5 hour \\
$437 \mathrm{~K} /$ minute & 1 hour & 3 hour & 5 hour \\
$507 \mathrm{~K} /$ minute & 1 hour & 3 hour & 5 hour \\
\hline
\end{tabular}

of the average precipitate size and its distribution. The samples were prepared by cutting slices normal to the solidification direction, mounting, grinding with progressively smaller grit size and final polishing with aluminium oxide suspension with grit size of $0.02 \mu \mathrm{m}$. Chemical etching was performed using Kalling's solution which preferentially removes the $\gamma^{\prime}$ phase in order to enhance the contrast of precipitate/matrix interface. The SEM micrographs were analysed using the freely available image processing software ImageJ following methods reported in [7]. Each image in grayscale was imported into the software and converted to binary using a threshold value, to distinguish matrix from precipitate. The software was then calibrated using the scale bar of the image; thereafter a particle search was performed to return a list of the particles found together with their respective areas. The size of the particle was taken as the diameter of a circle of area equivalent to that determined. For each sample a minimum of 5 images were analysed and the average value of the diameter taken. A micrograph of the as-received material is given in Fig. 10. It can be seen that the structure is pretty irregular; the $\gamma^{\prime}$ particles

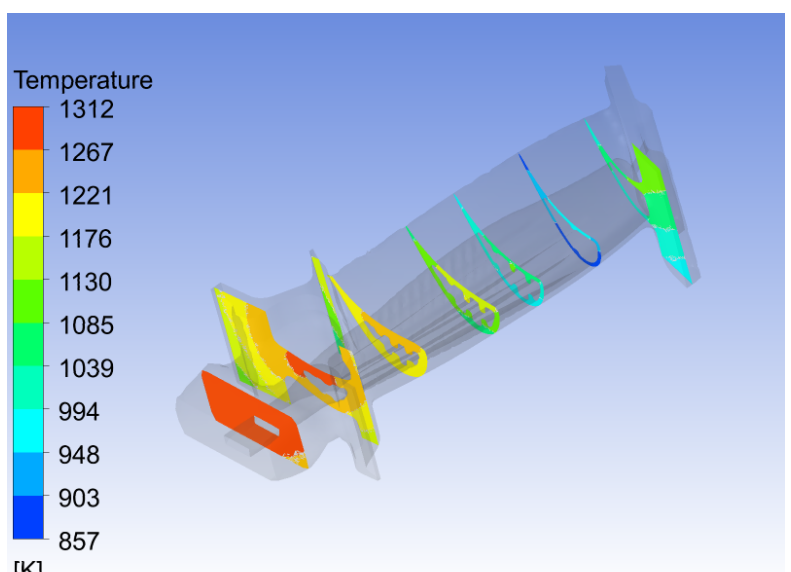

[K]

Figure 9: Predicted temperature within various sections of the blade.

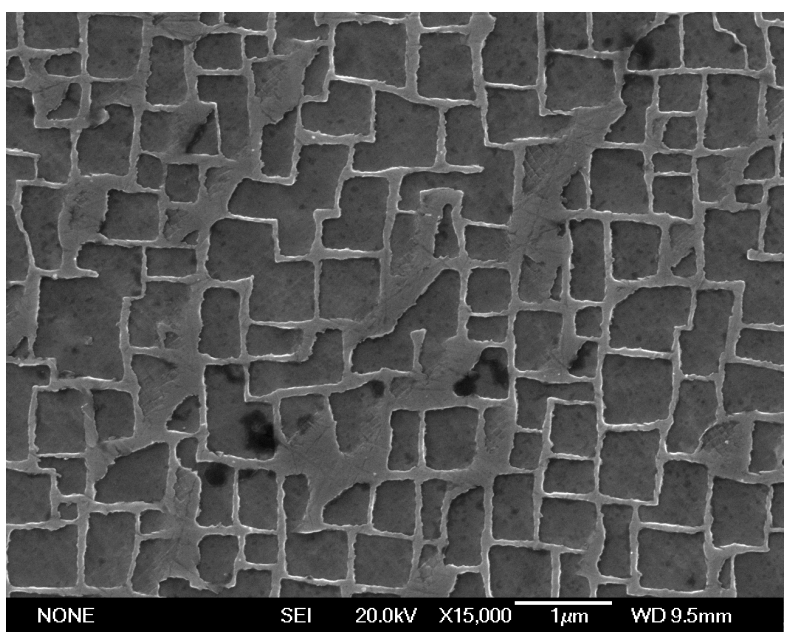

Figure 10: SEM micrograph of an RR3010 solution treated with a standard quench rate.

appear as approximately cubical but the edges are not sharp. This is typical of a structure that has been subject to a slow quenching. The average $\gamma^{\prime}$ size was found to be $538 \pm 81 \mathrm{~nm}$. In Fig. 11 the evolution of the structure during ageing is shown. The micrographs refer to the material quenched at 437 $\mathrm{K} /$ minute and its subsequent ageing.

During rapid quenching small $\gamma^{\prime}$ particles precipitate with a very irregular shape. In the subsequent ageing heat treatment, the size increases sharply during the first hour and the shape alters towards cubic; thereafter the size evolves more slowly but with an increasing tendency for the precipitates to align more strongly along the cube directions. In the final image 11-d - after 5 hours of ageing - one can see that the $\gamma$ channels have become visibly more narrow. Similar considerations apply to the samples quenched with different rates. The measured values of the precipitate size and their evolutions are shown in Fig. 12.

\section{Creep Tests}

A matrix of creep tests was performed to investigate the creep performances of RR3010 with different average $\gamma^{\prime}$ size. From the results of the analysis of Fig. 12 two conditions were selected to produce material with a $\gamma^{\prime}$ size of $300 \mathrm{~nm}$ (a fast quench with short ageing time) and $500 \mathrm{~nm}$ (a slow quench with longer ageing time). The letter $\mathrm{F}$ (fast) and $\mathrm{S}$ (slow) will be used to refer to those. Tests were performed at three temperature, namely $1123 \mathrm{~K}\left(850{ }^{\circ} \mathrm{C}\right), 1223 \mathrm{~K}$ 


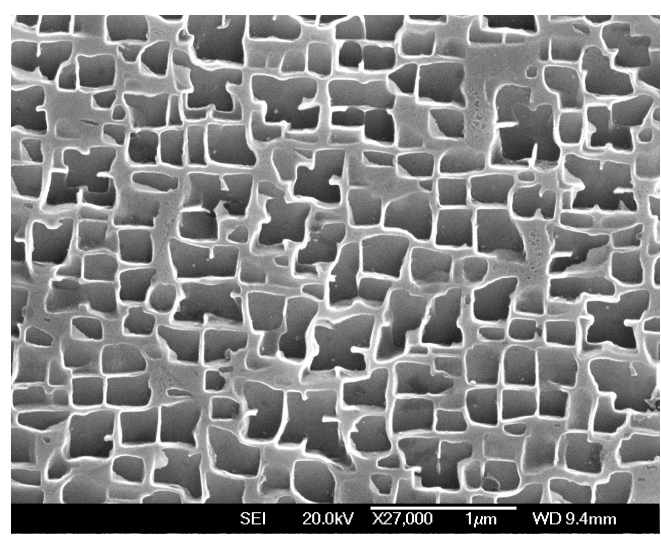

(a) RR3010 as quenched at $437 \mathrm{~K} /$ minute

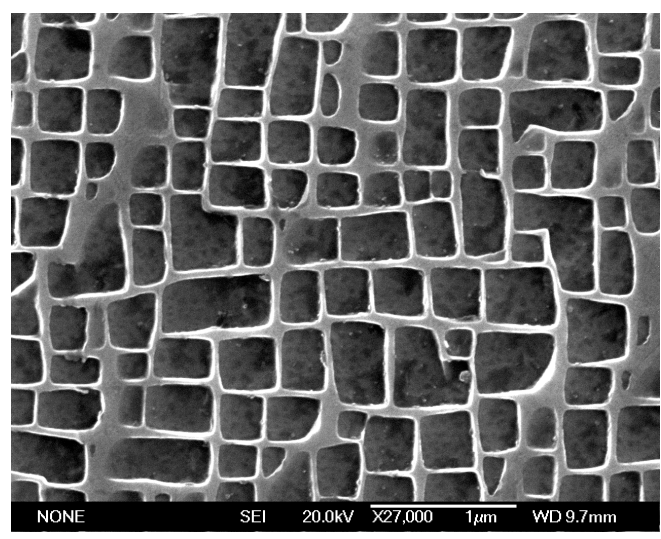

(c) After 3 hours of isothermal ageing

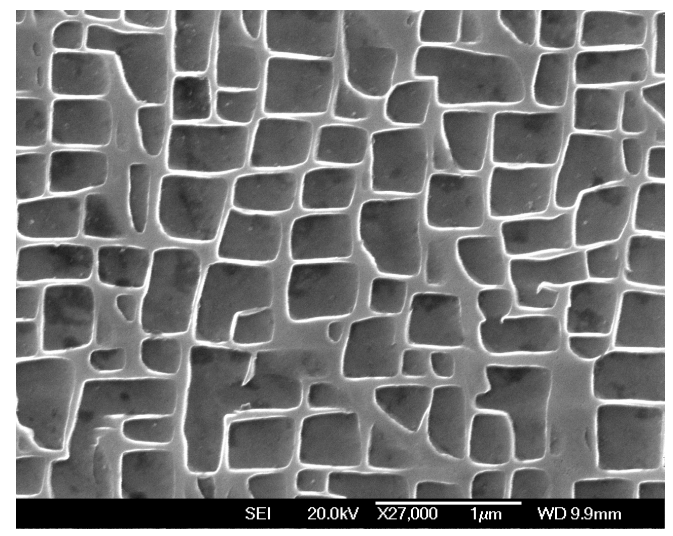

(b) After 1 hour of isothermal ageing

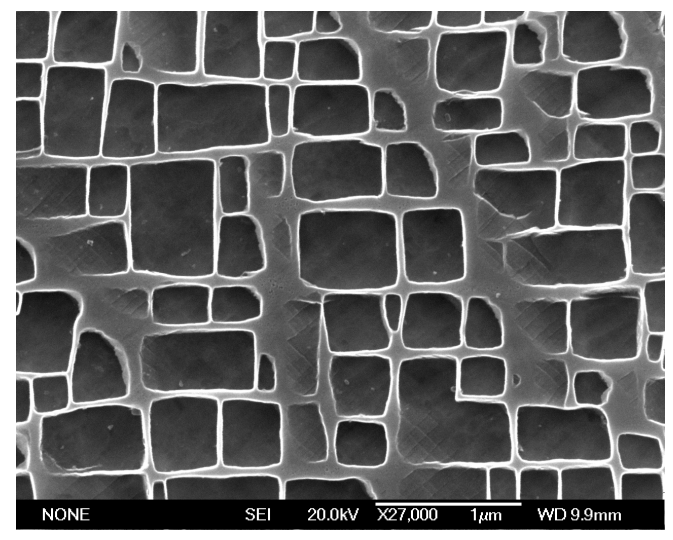

(d) After 5 hours of isothermal ageing

Figure 11: Evolution of precipitate microstructure with isothermal ageing at $1423 \mathrm{~K}\left(1150{ }^{\circ} \mathrm{C}\right)$

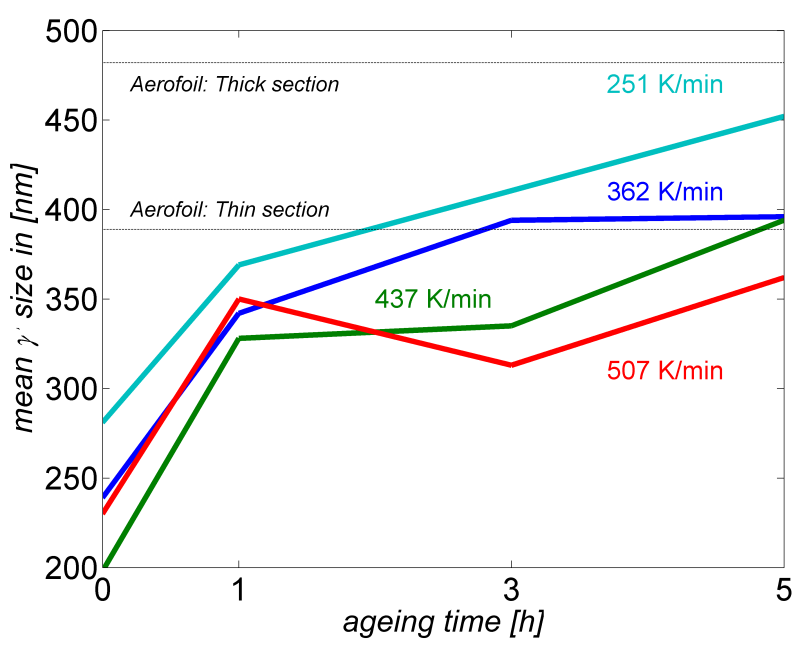

Figure 12: Evolution of average $\gamma^{\prime}$ size with ageing time. For comparison, the $\gamma^{\prime}$ size in different sections of a turbine blade is shown (horizontal lines). $\left(950{ }^{\circ} \mathrm{C}\right)$ and $1373 \mathrm{~K}\left(1100{ }^{\circ} \mathrm{C}\right)$ where the stresses were chosen as to achieve a rupture life of 100,300 and 1000 hours. The results of these tests are reported in Fig. 13. In the low temperature range the material $\mathrm{F}$, has a shorter life. In all cases it shows a much more pronounced primary creep than material S, while the secondary and tertiary regimes are almost similar. As the temperature increases, and the stresses decrease, the difference in mechanical properties between the two materials decrease. This is accompanied by a change in the shape of the creep curve, which implies a different mechanism for the deformation. At $1223 \mathrm{~K}$ $\left(950{ }^{\circ} \mathrm{C}\right)$ the primary creep is observed for very small strains (not at all at $330 \mathrm{MPa}$ ), and the performance of material S are always better. At 1373 K (1100 ${ }^{\circ} \mathrm{C}$ ) primary creep is completely absent, replaced by the tertiary creep regime where the strain rate increases monotonically until rupture; in this case the behaviour is almost identical. 


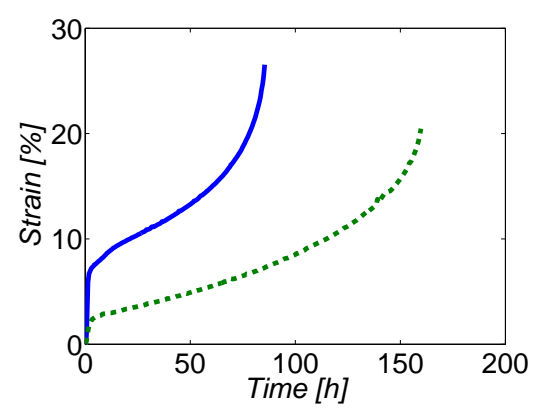

(a) $1123 \mathrm{~K}\left(850^{\circ} \mathrm{C}\right)-655 \mathrm{MPa}$

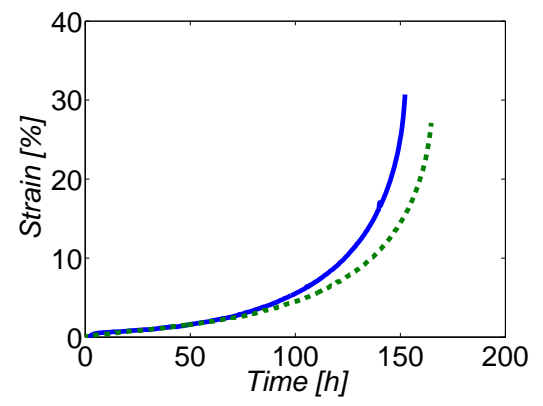

(d) $1223 \mathrm{~K}\left(950{ }^{\circ} \mathrm{C}\right)-435 \mathrm{MPa}$

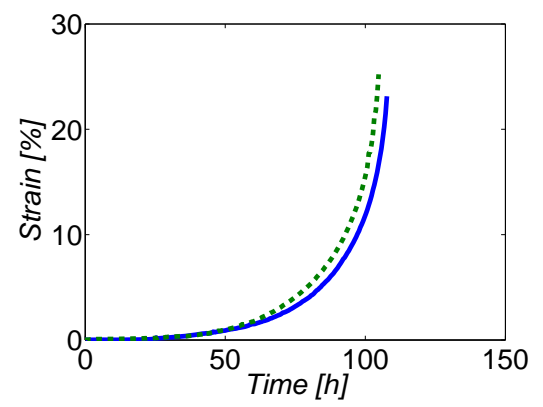

(g) $1373 \mathrm{~K}\left(1100{ }^{\circ} \mathrm{C}\right)-180 \mathrm{MPa}$

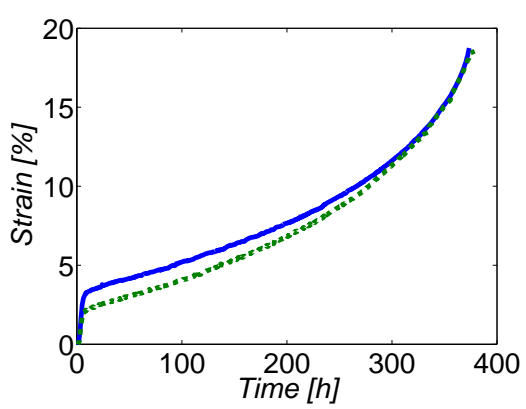

(b) $1123 \mathrm{~K}\left(850^{\circ} \mathrm{C}\right)-590 \mathrm{MPa}$

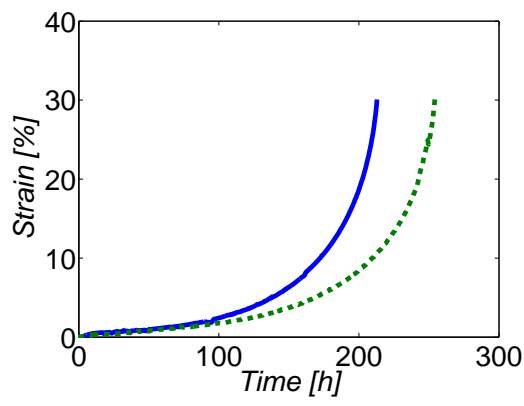

(e) $1223 \mathrm{~K}\left(950{ }^{\circ} \mathrm{C}\right)-400 \mathrm{MPa}$

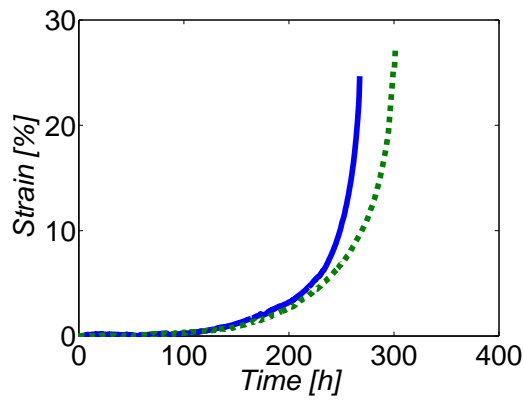

(h) $1373 \mathrm{~K}\left(1100{ }^{\circ} \mathrm{C}\right)-145 \mathrm{MPa}$

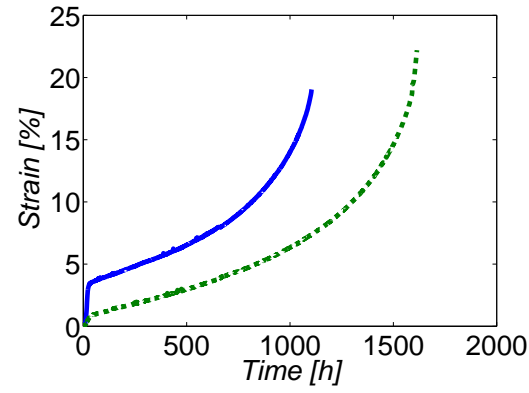

(c) $1123 \mathrm{~K}\left(850^{\circ} \mathrm{C}\right)-520 \mathrm{MPa}$

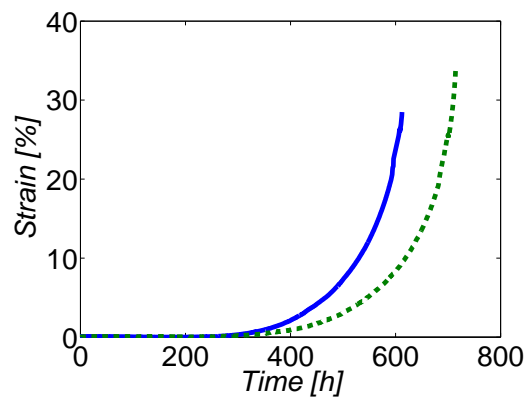

(f) $1223 \mathrm{~K}\left(950{ }^{\circ} \mathrm{C}\right)-330 \mathrm{MPa}$

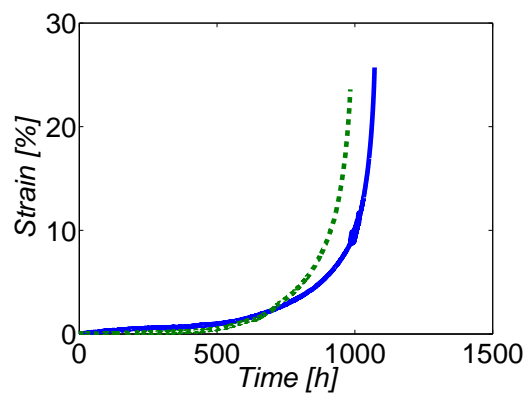

(i) $1100{ }^{\circ} \mathrm{C} 110 \mathrm{MPa}$

Figure 13: Creep test results. In each plot the curve relative to the fast quench (solid line) and slow quench (dashed line) are reported.

\section{Discussion}

The work reported in this paper shows how CFD simulations can be used to analyse the heat treatment process. Using information of heating element temperature and pressure drop across the furnace, the model predicts the temperature distribution inside the parts and the temperature history of the components including a prediction of local quench rate. Regions of high temperature, that can possibly lead to local melting, can be identified. The visualisation of the flow field shows the influence of the furnace geometry on the homogeneity of quenching, so that a more even cooling rate can be achieved across the fur- nace. This is fundamental to ensure that a uniform microstructure with uniform properties can be obtained. The microstructure is sensitive to the quench rate in terms of the precipitate size, as well as the order of the array of cuboids. It is shown how the ageing coarsens the structure and improves the alignment of the precipitates, reducing the $\gamma$ channel width. Fast quench used in this work generated very fine precipitate sizes. Even after ageing it was possible to retain a $\gamma^{\prime}$ size smaller than the one obtained in industrial processes. From the creep tests the effect of different structures on mechanical behaviour is evident. At low temperatures, primary creep deformation is due to the cutting of $\gamma^{\prime}$ particles, and therefore bigger 
precipitates are more resistent to cutting [8]. Once primary creep is over, the creep resistance of the two microstructures is similar. At intermediate and high temperature the deformation is controlled by dislocation activity and by the rafting phenomenon. At 1223 $\mathrm{K}\left(950{ }^{\circ} \mathrm{C}\right)$ microstructure $\mathrm{S}$ is stronger because the longer ageing time gives it a more ordered structure [9]. The F material despite having a smaller precipitate size, which should imply a smaller $\gamma$ channel width, is much more irregular so that regions of thick $\gamma$ are more common. Finally at $1373 \mathrm{~K}\left(1100{ }^{\circ} \mathrm{C}\right)$ the rafting regime is dominating the creep deformation and in this case one has to understand the effect of the initial $\gamma^{\prime}$ size on the raft formation. Nathal [10] found that an initially irregular structure forms wavy rafts with more terminations per unit area than an initially ordered array of precipitates. On the other hand in this work the difference in the initial structure is much less pronounced than in Nathal's work and therefore there is little difference.

\section{Conclusions}

The following conclusions can be drawn from this work:

1. Numerical modelling has been used to simulate the vacuum heat treatment and gas fan quenching used for the heat treatment of turbine blades using computational fluid dynamics.

2. The model captures correctly the physics involved and gives reliable predictions as shown by experiments. It is therefore suitable for the analysis of the heat treatment process and for future optimisation in simulation-driven process design.

3. Heating and quenching stages are simulated separately, to improve the numerical efficiency. During heating the component temperature is predicted, which allows homogeneity to be assessed and to identify possible locations of incipient melting. During quenching the cooling rate is estimated as a function of the furnace parameters. This allows the design of the furnace to be assessed.

4. The influence of the cooling rate on the structure and properties of the RR3010 superalloy has been investigated via SEM microscopy. The evolution of the microstructure with the ageing time has been characterised and its impact on creep performances has been studied.
5. The quenching technology used, allowed a wide range of precipitate sizes to be developed. Our data indicate that whilst the influence of particle size on creep deformation is fairly minor at temperatures above $1273 \mathrm{~K}$, at lower temperatures and especially when the $\gamma^{\prime}$ precipitates are subjected to shearing at high enough stresses, then larger $\gamma^{\prime}$ sizes confer better properties.

\section{Acknowledgements}

The authors are grateful to the Engineering and Physical Sciences Research Council (EPSRC) of the United Kingdom for funding under the SAMULET project. Rolls-Royce plc is acknowledged for the provision of the materials used for this study. The authors would like also to thank Dr. Atsushi Sato and Mr. Zailing Zhu for the useful discussions.

\section{References}

[1] G. E. Fuchs. Improvement of creep strength of a third generation, single-crystal Ni-base superalloy by solution heat treatment. Journal of materials engineering and performance, 11(1):19-25, 2002.

[2] N. Warnken, D. Ma, A. Drevermann, Roger C Reed, S.G. Fries, and I. Steinbach. Phase-field modelling of as-cast microstructure evolution in nickel-based superalloys. Acta Materialia, 57(19):5862-5875, November 2009.

[3] J. Svoboda, F. D. Fischer, P Fratzl, and Ernst Kozeschnik. Modelling of kinetics in multi-component multi-phase systems with spherical precipitates I : Theory. Materials Science and Engineering A, 385(1-2):166-174, 2004.

[4] Ernst Kozeschnik, J. Svoboda, P Fratzl, and F. D. Fischer. Modelling of kinetics in multi-component multi-phase systems with spherical precipitates:: II: Numerical solution and application. Materials Science and Engineering A, 385(1-2):157-165, 2004.

[5] Inc Ansys. ANSYS FLUENT Theory Guide. Knowledge Creation Diffusion Utilization, 15317(April):724-746, 2009.

[6] J.H. Ferziger and M Perić. Computational methods for fluid dynamics, volume 3. Springer Berlin etc, 1999.

[7] James Coakley, Hector Basoalto, and David Dye. Coarsening of a multimodal nickel-base superalloy. Acta Materialia, 58(11):4019-4028, 2010.

[8] M Maldini, H Harada, Y Koizumi, T Kobayashi, and V Lupinc. Tertiary creep behaviour of a new single crystal superalloy at $900 \mathrm{deg}$ C. Scripta Materialia(USA), 43(7):637-644, 2000.

[9] Pierre Caron and T Khan. Improvement of creep strength in a nickel-base single-crystal superalloy by heat treatment. Materials Science and Engineering, 61(2):173-184, 1983.

[10] M V Nathal. Effect of initial gamma prime size on the elevated temperature creep properties of single crystal nickel base superalloys. Metallurgical Transactions A, 18(11):1961-1970, 1987. 